\title{
BMJ Open Risk of dementia after Parkinson's disease in Taiwan: a population-based retrospective cohort study using National Health Insurance claims
}

\author{
Chih-Ching Liu, ${ }^{1}$ Yu Sun, ${ }^{2}$ Pei-Chen Lee, ${ }^{3}$ Chung-Yi Li, ${ }^{1,4}$ Susan C Hu ${ }^{\oplus}$
}

To cite: Liu C-C, Sun Y, Lee P-C, et al. Risk of dementia after Parkinson's disease in Taiwan: a populationbased retrospective cohort study using National Health Insurance claims. BMJ Open 2019;9:e025274. doi:10.1136/ bmjopen-2018-025274

- Prepublication history for this paper is available online. To view these files, please visit the journal online (http://dx.doi org/10.1136/bmjopen-2018025274).

\section{$\mathrm{C}-\mathrm{YL}$ and $\mathrm{SCH}$ contributed} equally.

Received 10 July 2018 Revised 8 December 2018 Accepted 17 December 2018

Check for updates

(C) Author(s) (or their employer(s)) 2019. Re-use permitted under CC BY-NC. No commercial re-use. See rights and permissions. Published by BMJ.

${ }^{1}$ Department of Public Health, College of Medicine, National Cheng Kung University, Tainan, Taiwan

${ }^{2}$ Department of Neurology, En Chu Kong Hospital, New Taipei City, Taiwan

${ }^{3}$ Department of Health Care Management, National Taipei University of Nursing and Health Sciences, Taipei, Taiwan

${ }^{4}$ Department of Public Health, College of Public Health, China Medical University, Taichung, Taiwan

Correspondence to

Dr Susan C Hu;

shuhu@mail.ncku.edu.tw

\section{ABSTRACT}

Objectives A nationwide cohort study on the risk of dementia onset after first diagnosis of Parkinson's disease (PD) is lacking. This study aims to assess 11 years of incidence and the HRs for developing dementia in patients with PD compared with matched controls.

Design A population-based cohort study.

Setting National Health Insurance database in Taiwan. Participants A total of 5932 patients with PD were identified, and 29645 age-matched, sex-matched and index year-matched PD-free individuals were randomly selected.

Outcome measures All subjects were linked to the claim data to identify the first diagnosis of dementia. The Poisson assumption was used to estimate the incidence rate. Cause-specific hazards models with a partitioning of time at 1 year to account for proportionality were used to estimate the risk of dementia onset.

Results The median duration from the first diagnosis of PD to the development of dementia was 9.02 years. In the first partition ( $\leqq 1$ year), the incidence of dementia in the PD and control groups was 114.49 and 9.76 per 1000 person-years, respectively, with an adjusted HR of 6.43 (95\% Cl 5.46 to 7.57 ). In the second partition (>1 year), the incidence of dementia in the PD and control groups was 30.99 and 10.83 per 1000 person-years, with an adjusted HR of 2.42 (95\% Cl 2.23 to 2.61). Notably, in the second partition, both men and women aged $<70$ years had the highest $\mathrm{HR}(3.82,95 \% \mathrm{Cl} 2.79$ to 5.22 and $4.27,95 \% \mathrm{Cl}$ 3.25 to 5.63 , respectively).

Conclusions This study noted an increased risk of dementia after a diagnosis of PD. The magnitude of effect estimation was higher in men in the first partition but was similar in both genders in the second partition. PD patients aged $<70$ years have the highest risk of dementia in any given partition time.

\section{INTRODUCTION}

Dementia, a symptom of cognitive disturbances, can be potentially disabling and can also be related to increased mortality rates and costs. ${ }^{2}$ Thus, information about which patients will eventually develop dementia is an important issue in public health and clinical practice. ${ }^{3}$

\section{Strengths and limitations of this study}

- The study strengths include the fact that it is a nationwide, retrospective cohort design for 11 years with more accurate estimates of the incidence rates of dementia by using the first diagnosed PD cases rather than the prevalent cases as study subjects.

- A multivariate Cox proportional hazard regression with a competing risk analysis was used to control the confounding bias and account for the competing risk of death.

- We were unable to consider a comprehensive list of potential confounders, such as smoking, educational level, physical function and genes in the analysis because of the limited information available from the claims data.

- Another limitation is the lack of clinical symptoms and subtypes of dementia.

Parkinson's disease (PD) has been associated with the development of cognitive impairment. ${ }^{4}$ However, most previous studies on the association between PD and dementia risk have been conducted in western countries, ${ }^{5-19}$ and information for Asian PD populations is lacking. Moreover, to identify robust HRs of dementia in PD requires a large sample size cohort and a sufficiently long follow-up time to observe the development of symptoms of dementia in incident cases of PD.

To the best of our knowledge, there has been no whole population-based nationwide cohort study on this topic, ${ }^{5-23}$ and only few cohort studies have involved incident cases of PD to investigate the frequency of dementia with a follow-up period of more than 10 years. The Sydney Multicentre Study of PD followed 136 newly diagnosed patients with PD more than 20 years and reported that $83 \%$ of the 30 survivors developed dementia. However, only PD cases who received low-dose levodopa or low-dose bromocriptine were included in this study, which may not represent PD within the 
population as a whole. ${ }^{19}$ The CamPaIGN study followed 121 newly diagnosed PD cases for 10 years, of which 41 PD cases developed dementia. This study estimated dementia incidence in PD subjects was 54.7 per 1000 person-years (95\% CI 35.4 to 74.1 ), which was 2.6-fold higher than that in an age-matched and geographically matched population. However, this study also only included only a few newly diagnosed PD cases $(\mathrm{n}=121) .^{9}$

Moreover, many studies have included prevalent PD cases at varying disease stages to investigate the risk of dementia, which may have caused survival bias. ${ }^{6-8} 1012-151718202223$ For example, the Rotterdam study recruited 72 prevalent and 67 incident PD cases with only an overall mean follow-up time of 6.9 years and found a positive association between PD and dementia incidence. ${ }^{14}$ In Taiwan, the only population-based study with a case-control design also showed a positive association between PD and the risk of dementia. ${ }^{22}$ However, potential survival bias resulting from recruitment of prevalent PD cases at various disease stages may have been present in these studies.

Some studies have reported that older age $e^{5-10} 13152023$ and male gender ${ }^{5}$ are related to increased dementia risk in PD; however, information regarding the age-stratified and sex-stratified dementia incidence rate in PD is scant. In addition, many patients with PD may have medical comorbidities such as stroke, hypertension, diabetes mellitus, hyperlipidaemia and coronary heart disease, which may have modification effects on the relationship between dementia and PD. ${ }^{76} 1722$ However, little research has examined medical comorbidities as a potential confounding factor that should be controlled for. ${ }^{722} 23$ Moreover, because of the increased age and comorbidities in a long-term follow-up study, competing risk of death should be considered. However, none of studies on this topic have considered death as a competing risk, ${ }^{6-2123}$ which may induce potential attrition bias and tend to distort the study results.

Given the above-mentioned methodological problems and limited information on this topic, the association between PD and the risk of dementia needs to be further explored. Therefore, in this study, a nationwide population-based cohort study was conducted to estimate 11 years of incidence and the HRs for development of dementia in patients with first-diagnosed PD by age and sex and by comorbidities (ie, hypertension, diabetes, coronary artery disease $[\mathrm{CAD}]$, stroke, hyperlipidaemia and chronic obstructive pulmonary disease [COPD]), after accounting for the competing risk of death.

\section{METHODS}

\section{Data source}

The dataset was drawn from ambulatory care claims, inpatient claims and the updated registry for beneficiaries retrieved from Taiwan's National Health Insurance Research Database (NHIRD), as provided by the National Health Insurance Administration (NHIA), Ministry of
Health and Welfare, Taiwan. A universal National Health Insurance (NHI) programme has been implemented in Taiwan since 1995, and more than 99\% of Taiwan residents enrolled in the NHI programme after 2000. The NHIA has contracts with $92.5 \%$ of the hospitals and clinics in Taiwan. ${ }^{24}$ The NHIA performs quarterly expert reviews on a random sample of every 50-100 ambulatory and inpatient claims in each hospital and clinic to ensure the accuracy of the claims data. ${ }^{24}$ Therefore, information obtained from the NHIRD is considered to be complete and accurate. The NHI datasets have been used in many published epidemiological studies on $\mathrm{PD}^{2526}$ and dementia. $^{27}$

\section{Patient and public involvement}

We conducted this study by using the NHIRD. No patients or members of the public were involved in the development of the research question and outcome measures. Also, no patients or members of the public were involved in setting out the design of this study, nor were they involved in the recruitment of and conducting of the study. The study results were not disseminated to the study subjects.

\section{Study design, cohorts and covariates}

This was a retrospective cohort study from 2002 to 2012. We selected 5932 eligible patients with PD between 2002 and 2003 from a previous study for which sample selection details were discussed previously. ${ }^{28}$ In brief, the PD cohort in this study included all cases with at least three medical claims (either outpatient or inpatient care) with a diagnostic code of PD (International Classification of Diseases, 9th Revision, Clinical Modification [ICD-9-CM] code 332.0) who are receiving at least three times of prescriptions of anti-Parkinsonism medications, including L-dopa or dopamine agonist prescriptions after a firsttime diagnosis between 2002 and 2003. Moreover, the first and last outpatient or inpatient visits and anti-Parkinsonism medication records were separated by at least 90 days to avoid accidental inclusion of miscoded patients.

To ensure that the PD diagnosis was reliable and consistent, cases were excluded if: (1) an age on the index date of less than 40 years who are more likely to have a genetic aetiology; (2) a diagnostic code of secondary Parkinsonism (ICD-9-CM code: 332.1) during the study period; (3) receipt of any neuroleptic medication 180 days prior to the index date; and (4) three or more medical claims (either ambulatory or inpatient care) with diagnostic codes of dementia prior to the index date. The first date of initial diagnosis of PD in the period of 2002 to 2003 was set as the index date.

We previously conducted a pilot study to validate the accuracy of the ICD-9 coding in patients with PD. ${ }^{28}$ In this study, medical records including symptoms/signs, diagnostic procedures, use of antiparkinsonism medication, as well as response to medication of 290 randomly selected patients with ICD-9-CM coded 332.0 were examined in detail by three experienced neurologists from 
January to October 2012. Among these 290 cases, 245 were confirmed as PD patients based on the aforementioned clinical information. The sensitivity, specificity, positive predictive value and negative predictive value were $97.6 \%, 92.3 \%, 98.8 \%$ and $85.7 \%$, respectively. The accuracy of our method for identifying PD cases was $96.9 \%$. Moreover, cases in this study were ascertained using the ICD code and required having been prescribed at least three times of antiparkinsonism medication including L-dopa or a dopamine agonist to minimise the possibility of miscoding.

The control subjects were selected from those who had not been diagnosed with PD or treated with any anti-PD medications during the entire study period and met the same exclusion criteria as those set for the patients with PD. These control subjects were matched by age (each 5 year span), sex and year of index date for patients with $\mathrm{PD}$ at a 5:1 ratio. As a result, 29645 control subjects were identified. For the control groups, the index date was either 1 January 2002 or 1 January 2003.

Baseline comorbidities that may be associated with an increased risk of dementia were identified for the PD and control groups. These included hypertension, diabetes, CAD, stroke, hyperlipidaemia and COPD observed before the index date. The comorbidity score observed before the index date was calculated using the Charlson comorbidity index, which is a weighted summery measure of common comorbid conditions adopted for use with ICD-9-CM-coded administrative databases. ${ }^{29-31}$ Information on the geographic area, urbanisation level, occupational status and salary-based insurance premium at the index date was also obtained from the registry for beneficiaries. The number of medical visits within 1 year after the index date was adjusted to decrease the potential presence of surveillance bias because subjects with $\mathrm{PD}$ visit clinics more frequently and thus may have more opportunities to be diagnosed as having dementia.

\section{End point and statistical analysis}

The main outcome variable was the initial occurrence of dementia (ICD-9-CM code: 290, 294.1, 331.0 and 331.82). A study in Taiwan has previously reported that the diagnostic accuracy of dementia is approximately $90 \%$ when relying on diagnosis codes (ICD-9-CM) to identify dementia. ${ }^{27}$ To increase the validity of dementia identification, only dementia cases diagnosed with $\geqq 3$ ambulatory visits or $\geqq 1$ hospitalisation were included in this study. We did not distinguish the subtypes of dementia because of data limitations due to a lack of information regarding symptoms/signs, lab data and image findings, and further outcome analyses with dementia subtype classifications, such as dementia with Lewy bodies (DLB), Alzheimer's dementia, frontotemporal dementia and just Parkinson's disease dementia (PDD), were not performed. However, according to the criteria set forth by the consensus report of the Lewy Body Consortium, ${ }^{32}$ clinicians and researchers use the 'one year rule' to help verify the diagnoses of DLB and PDD. Thus, we analysed the dementia incidence within and after 1 year of PD diagnosis, respectively.

We followed the study subjects from the index date to the first diagnosis of dementia, withdrawal from the NHI or 31 December 2012, whichever came first. The incidence density of dementia was calculated using an age-specific and sex-specific and comorbidity-specific stratified analysis based on the Poisson assumption. The cumulative events and rates of dementia according to the PD status over the study period were calculated using a Kaplan-Meier analysis, and the log-rank test was used to test the between-group differences.

Since death is the competing risk of dementia occurrence in this long-term follow-up study, analytical approaches used in competing risk settings must be used to assess the association between PD and the risk of dementia. Cause-specific hazards models, one of the most common analytical methods used in competing risk settings, are better suited for studying the aetiology of diseases. ${ }^{33}$ The cause-specific hazard is the instantaneous risk of dying from a particular cause $\mathrm{k}$ given that the subject is still alive at time t. ${ }^{34}$ Thus, in this study, a Cox proportional hazard regression with competing risk models, according to cause-specific hazards models, was performed to assess the HR of dementia in relation to PD.

In addition, we performed a sex-stratified and age-stratified analysis and a comorbidity-stratified analysis to examine the potential effect modifications by age, sex and comorbidity on the association between PD and the risk of dementia. Plots of $\log (-\log$ (survival function) ) vs $\log$ (time) were drawn to test for violations of the proportional hazards assumption. Therefore, separate time-partitioned models were created, and the hazards within each partition were assessed. Proportionality was held for the new models partitioned at 1 year. If we modelled the hazards for $\leq 1$ year (ie, the first time partition), the censoring day for subsequent events was 1 year. If we modelled the hazards for $>1$ year (ie, the second time partition), subjects with earlier events were included and considered to be censors (because the exclusion of these subjects may lead to a survival bias). A $p<0.05$ was considered significant.

\section{RESULTS}

The distributions of age and gender were no significant difference in both groups. The percentages of patients with PD who lived in urban $(54.7 \%$ vs $51.8 \%)$ and suburban $(35.6 \%$ vs $33.2 \%)$ areas and in northern $(45.6 \%$ vs $44.8 \%)$ and central $(25.5 \%$ vs $24.9 \%)$ Taiwan were higher than those of the controls. The prevalence rates of the risk factors for dementia were high in patients with PD. The PD cohort had fewer white-collar workers (25.0\% vs $31.2 \%, \mathrm{p}<0.0001)$, a lower insurance premium (percentage with none or a lower than median insurance premium: 69.5 vs $60.0, \mathrm{p}<0.0001$ ), a higher Charlson's score (percentage with score of 1 to $\geqq 2: 52.1 \%$ vs $25.4 \%, \mathrm{p}<0.0001)$ and a higher frequency of medical visits 
(39.6 vs 21.9 per year, $\mathrm{p}<0.0001$ ), than the control group (table 1).

Figure 1 shows the cumulative incidence of dementia in patients with and without PD. The cumulative incidence of dementia for PD was significantly higher than the corresponding data observed in the non-PD group (log-rank test, $\mathrm{p}<0.0001$ ).

Among the total of 5932 first diagnosed PD cases, only 492 of these cases $(8.3 \%)$ were derived from inpatient records. The adjusted HRs of dementia either in the overall PD cases or in the PD cases only enrolled in an outpatient group were significantly higher than those in the control group without PD. The median duration from the overall first diagnosis of PD to the development of dementia was 9.02 years.

During the 11 years of follow-up, a total of 1836 patients with PD developed dementia, and 1226 patients with PD died without developing dementia. In the same period, a total of 3159 control subjects developed dementia, and 5223 control subjects died without developing dementia. In the period within 1 year after the index date (ie, the first time partition), a total of 5932 PD subjects encountered 624 medical episodes due to first diagnosed dementia in the 5450.09 person-years observed, representing incidence densities of dementia of 114.49 per 1000 person-years. In the same period, a total of 29645 PD subjects encountered 285 medical episodes due to first diagnosed dementia in 29208.39 person-years observed, representing incidence densities of dementia of 9.76 per 1000 person-years. Noticeably, the incidence density of dementia increased with age irrespective of PD status and sex, and the highest incidence was observed in those aged $\geqq 80$ years. The adjusted HR of dementia in relation to PD was significantly increased at 6.43 (95\% CI 5.46 to 7.57 ) and was higher in men than in women (HR: $7.18,95 \%$ CI 5.73 to 9.01 vs $5.54,95 \%$ CI 4.39 to 6.99 ). In addition, there was a significant interaction of PD with age on the risk of dementia for both men $(p=0.02)$ and women $(\mathrm{p}<0.0001)$ (table 2$)$.

In the years following the PD diagnosis (ie, the second time partition), the overall incidence density of dementia was much lower (table 2). The change in incidence density between the first and the second partition was more pronounced in the PD group (from 114.49 to 30.99 per 1000 person-years) than in the control group (from 9.76 to 10.83 per 1000 person-years). The age-specific and sex-specific incidence densities had a similar pattern in terms of change. However, no significant difference in the sex-specific HRs of dementia was observed ( $p=0.23)$. There was a significant interaction of PD status with age $(p<0.0001)$ in both sexes. Further analyses of age-specific and sex-specific HRs showed the highest HR was observed in women with PD aged $<70$ years (HR: 4.27 ; 95\% CI 3.25 to 5.63$)$.

Impact of PD on the risk of dementia by comorbidity is shown in table 3. Irrespective of the various time partitions, the incidence of dementia increased with the number of comorbidities in both groups. The PD group had the highest risk of dementia across various medical comorbidity stratifications or Charlson's scores after adjusting for baseline characteristics. In the first time partition, the interaction of $\mathrm{PD}$ with hypertension $(p=0.01)$, CAD $(p=0.02)$, stroke $(p<0.0001)$ and COPD $(p=0.04)$ on the risk of dementia also was statistically significant, indicating that subjects without hypertension, CAD, stroke and COPD had a higher adjusted HR for dementia. However, although the adjusted HR for dementia was also higher in subjects without diabetes and hyperlipidaemia than in those with medical comorbidities, there was no statistically significant modification effect by diabetes and hyperlipidaemia on the association between PD and the risk of dementia. In terms of the Charlson's scores, subjects with scores of 0 had a higher adjusted HR for dementia than those with scores of 1 and $\geqq 2$. The interactions were significant for PD with Charlson's score $(p=0.01)$ on the risk of dementia.

In the second time partition, effect modification by hypertension $(\mathrm{p}<0.0001), \mathrm{CAD} \quad(\mathrm{p}=0.01)$ and stroke $(p<0.0001)$ was statistically significant for dementia, indicating that subjects without those medical comorbidities had a higher adjusted HR for dementia. Among patients with diabetes, hyperlipidaemia or COPD, adjusted HR for dementia also showed a statistically significant high risk from $2.02(95 \%$ CI 1.68 to 2.43$)$ to 2.21 (95\% CI 1.89 to 2.59 ), but no significant modification effect was found for those with medical comorbidities on the association between PD and the risk of dementia. In terms of the Charlson's scores, subjects with scores of 0 had a higher adjusted HR for dementia than those with scores of 1 and $\geqq 2$. Also, a significant modification effect of Charlson's scores on the association between PD and the risk of dementia $(p=0.01)$ was found. Regardless of whether medical comorbidities existed or not, the HRs for dementia were greater in the first time partition but were smaller in the second time partition.

\section{DISCUSSION}

To the best of our knowledge, this is the first nationwide population-based cohort study to demonstrate that patients with the first diagnosis of PD are associated with increased risk of dementia compared with non-PD patients. However, our study showed a sharply increased hazard of dementia within 1 year after the first diagnosis of $\mathrm{PD}$, which is clinically and biologically questionable, probably because a large proportion of patients with dementia remain undiagnosed before the index date of their first clinical visit for PD.

In other words, many patients with pre-existing cognitive impairment and PD were classed as new PD cases because their condition had deteriorated and had only been recognised because they had been referred for an expert opinion. This is also supported by the decline in the number of PD patients with dementia in the subsequent period of observation. In this study, we found that the overall risk of dementia onset increased nearly 
Table 1 Characteristics of the study subjects

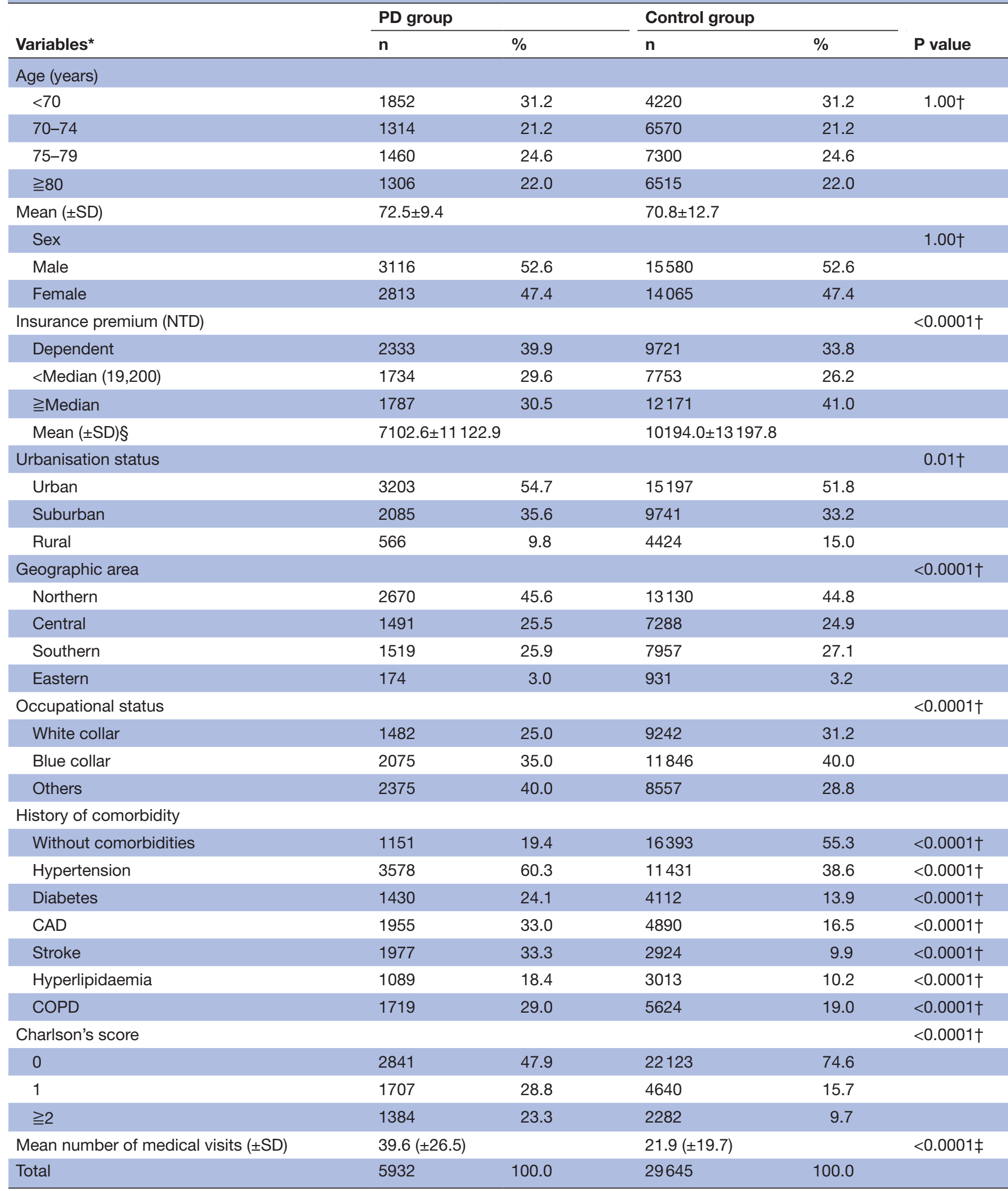

*Inconsistency between the total population and the population summed for individual variables was due to missing information. †Based on $\chi^{2}$ test.

$\ddagger$ Based on a Student's t-test.

$\S$ The dependent insurers were not included.

CAD, coronary artery disease; COPD, chronic obstructive pulmonary disease; NTD, New Taiwan dollars. 


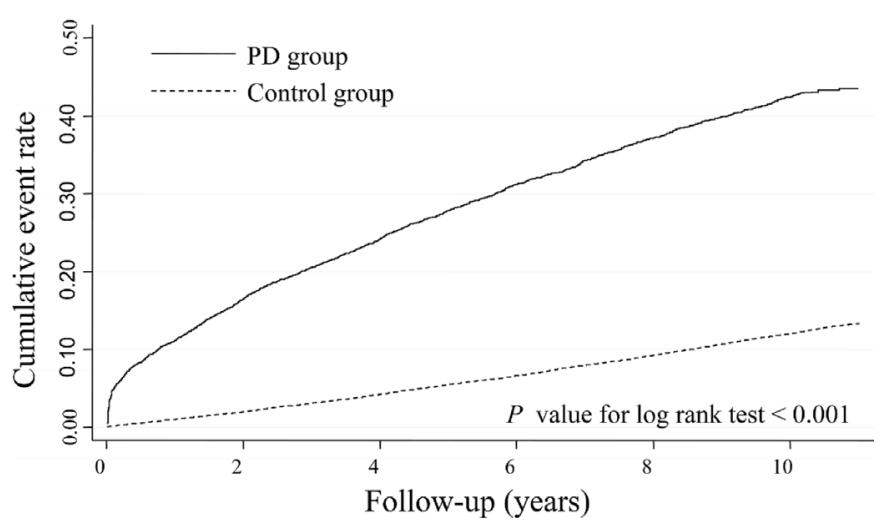

Figure 1 Comparison of Kaplan-Meier failure estimates of dementia onset between the two groups. PD, Parkinson's disease.

twofold in up to 11 years (adjusted HR: 2.42, 95\% CI 2.23 to 2.61) among those who survived at least 1 year and had an initial PD diagnosis thereafter. The magnitude of this association varied according to different age and sex stratifications. In general, the increased risk of dementia was higher in men in the first partition but was similar in both genders in the second partition. In addition, younger patients with PD have the highest risk of dementia in any given partition time. The study results can provide physicians and patients with valuable information and also demonstrate the need for guidelines for detection of dementia risk after the initial diagnosis of PD.

Our study shows that a reasonably increased hazard of dementia more than 1 year after diagnosis of PD is more likely to be real and may suggest evidence of the mechanisms supported by the Braak pathology staging hypothesis. ${ }^{35} 36$ Our findings were similar to another population-based case control study in Taiwan, ${ }^{22}$ which showed the risk of developing dementia in prevalent Parkinsonism was highest in the first 6 months (adjusted OR (AOR): $11.98,95 \%$ CI 8.51 to 16.68 ) and then became lower in the following months to years after diagnosis.

Age is known to be a risk factor for dementia in the general population ${ }^{37} 38$ and in the PD patient population. ${ }^{5-10} 13152023$ This may be caused by ageing of non-dopaminergic structures (ie, the locus ceruleus and pedunculopontine nucleus). ${ }^{39}$ However, a modifying effect of age on the risk of dementia after PD may be present in our study. For example, we found that patients with PD had a significantly higher overall risk of dementia than those in the control group, particularly in subjects aged $<70$ years. This result is similar to the findings of some prior studies. ${ }^{13} 18$

Male sometimes is identified as a risk factor for dementia in $\mathrm{PD}^{5}$; however, there is no clear explanation for this finding. In our study, we found that the risk of dementia was similar in both men and women who had first-diagnosed PD 1 year later (HR: 2.44, 95\% CI 2.19 to 2.73 and HR: $2.41,95 \%$ CI 2.15 to 2.69 , respectively). Accordingly, patients with PD, especially younger patients in both sexes, could be selected in future studies as a target population to evaluate whether interventions are effective in decreasing the risk of dementia after diagnosis of PD.

Our study also shows that the overall risk of dementia was more than double (adjusted HR 2.42) among subjects with first-diagnosed PD 1 year later for up to 11 years. After accounting for the competing risk of death and adjustment for the number of medical visits, the findings were similar to those of Perez et al, ${ }^{11}$ who reported a higher HR of incident dementia $(2.47,95 \%$ CI 1.55 to 3.95) in patients with PD as compared with non-PD subjects. However, other cohort studies have shown a HR ranging from 1.7 (95\% CI 1.1 to 2.7) to 5.9 (95\% CI 3.9 to 9.1) for incident dementia in PD groups compared with the general population, ${ }^{9} 1012-1518$ which is different from our findings. Noticeably, most previous studies were limited by a relatively small sample size, ${ }^{6-17} 19-2123$ shorter follow-up time, ${ }^{6-810} 12-1820$ the lack of a matched control, ${ }^{6-8161719-2123}$ failure to account for the competing risk of death ${ }^{6-21} 23$ or a lack of adjustment for the number of medical visits to control for surveillance bias, ${ }^{9-15} 18$ rendering the risk that the estimates were more likely to be imprecise and biased.

We found the incidence of dementia increased with the number of comorbidities, including hypertension, diabetes mellitus, $\mathrm{CAD}$, stroke, hyperlipidaemia and COPD, irrespective of PD status. However, in our study, the adjusted HR for dementia was higher in PD alone than in those with medical comorbidities. The effect modification by hypertension, CAD and stroke was statistically significant for the association between PD and dementia in any given partition time. Prior studies regarding the relationship between patients with PD and these comorbidities remain controversial. ${ }^{7} 161722$ For example, although a study in Taiwan demonstrated that patients with PD with cerebrovascular or cardiovascular comorbidities had a lower risk of dementia onset than patients with PD alone, ${ }^{22}$ which is similar to our findings, other studies have failed to find this relationship. ${ }^{16} 17$ In addition, some previous studies have shown that PD with cardiovascular dysautonomia (such as hypertension, diabetes mellitus and CAD) and COPD might cause substantial cerebral hypoperfusion and hypoxia, respectively. ${ }^{40-42}$ Hypoxia and hypotension in the brain might cause neuronal damage and increase accumulation of pathological proteins such as $\beta$-amyloid, which result in increased risk of dementia onset. ${ }^{40}{ }^{41}$ Therefore, future perspective studies focusing on the causal relationship between such comorbidities and the risk of dementia in PD are warranted.

There were several strengths in our study. First, we obtained a large, nationwide number of participants by using NHIR datasets, which made it possible to reduce selection bias, to obtain higher statistical power, to obtain a highly representative study population, to have a lower rate of non-response or loss to follow-up and to facilitate the age-stratified, sex-stratified and comorbidities-stratified analyses with an ample simple size to satisfy 


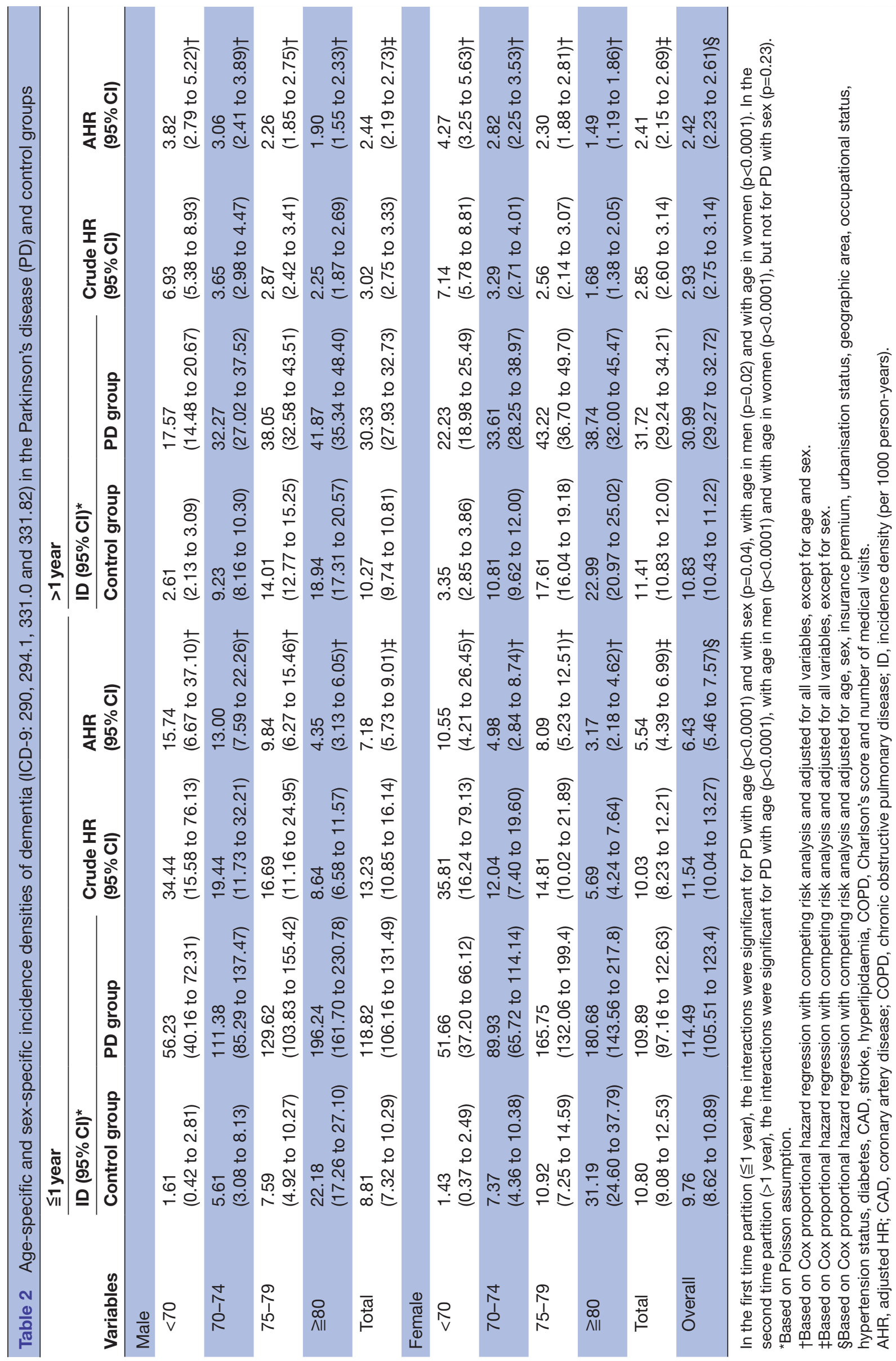




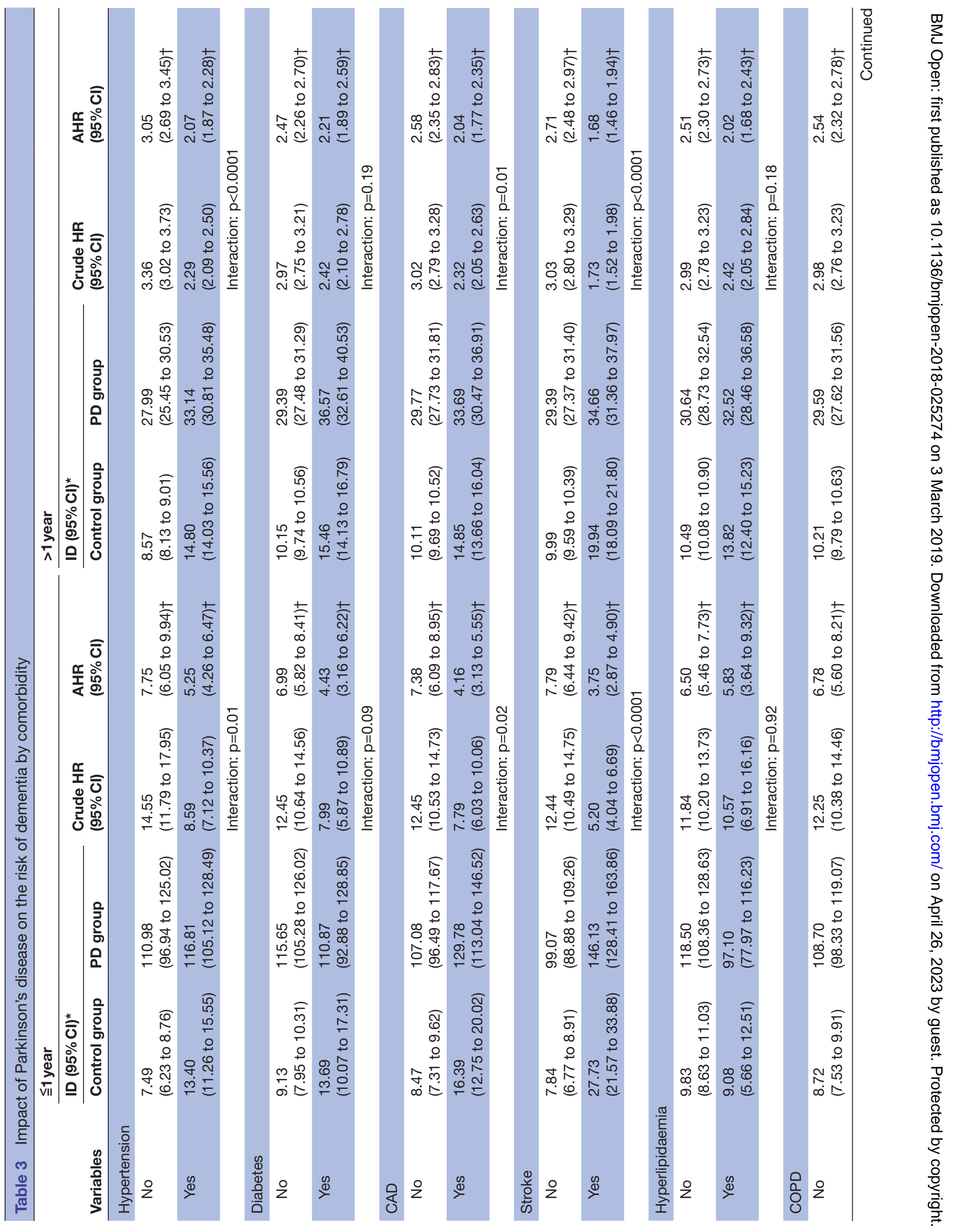




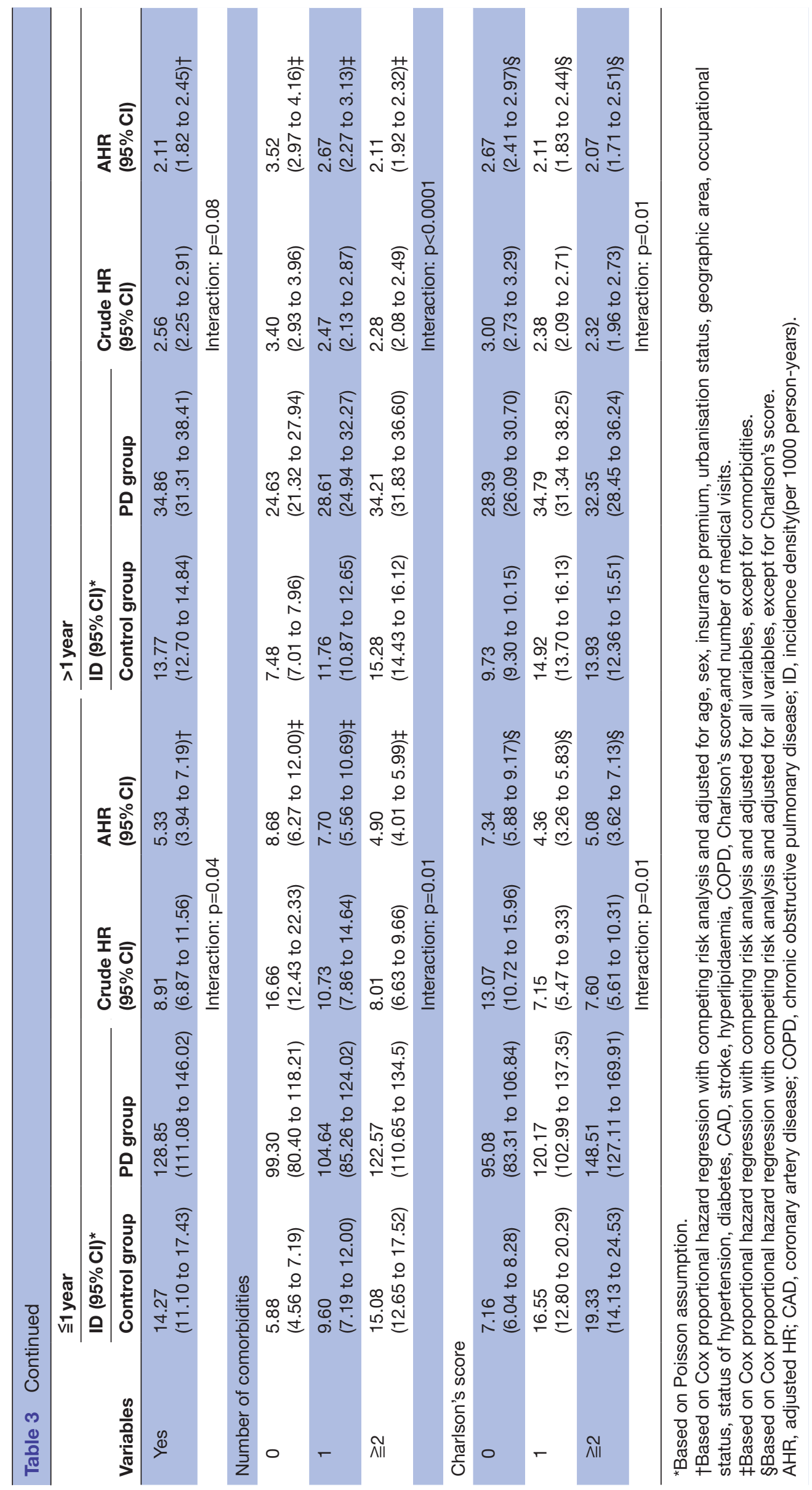


requirements. To the best of our knowledge, this study is the first to report the age-specific and sex-specific incidence rates of dementia in a PD group. Second, we conducted a longitudinal and retrospective cohort study for 11 years, which is a longer time during which to observe the development of dementia than that in many other prior studies. ${ }^{6-8} 10$ 12-18 20 Third, more accurate estimates for the incidence rates of dementia in the PD group are available in this study due to the usage of the first diagnosed PD cases rather than the prevalent PD cases, as this might reduce the variations in the incidence of dementia across various PD durations. Lastly, a multivariate Cox proportional hazard regression with a competing risk analysis was used to control for the confounding bias and to account for the competing risk of death.

Still, our study had some limitations. First, we solely selected our PD cases according to physician-recorded diagnosis and prescriptions reported in medical claims, which might have led to potential disease misclassification. However, we used at least three PD-related diagnoses and prescriptions, with the first and last visits $>90$ days apart, which greatly decrease the likelihood of disease misclassification. Similarly, because we selected patients with dementia only by using NHIR datasets, potential disease misclassification may be present. To address this concern, we only included dementia cases diagnosed with $\geqq 3$ ambulatory visits or $\geqq 1$ hospitalisation in this study to increase the validity of dementia identification.

Second, because patients with PD may use the healthcare system more often than control groups, surveillance bias may be present. Thus, to address this concern, we calculated the number of medical visits for 1 year after the index date and adjusted for this in the multivariate regression model. Also, the severity of dementia is not available in the database, and we could not distinguish subtypes of dementia in our datasets. Therefore, it is essential for patients with PD, particularly in high-risk groups such as subjects aged $<70$ years, to have regular cognitive assessments including combinations of neuropsychological markers throughout the early disease stages, which will provide benefits for identification of the subtypes in dementia and will decrease underestimation of risk for dementia in PD.

Third, due to the limited information available from the claims data, we were unable to consider a comprehensive list of potential confounders such as smoking, educational level, physical function and genes in the analysis, which may have resulted in residual confounding bias. To reduce such bias, we used COPD and occupational status as surrogates for smoking and educational level, respectively. Finally, the disease symptoms of PD cases at different disease stages may play a role, to some extent, in the relationship between PD and the risk of dementia. However, because information on the disease symptoms is unavailable from the NHI data, we have left this area (such as non-motor symptoms) for further investigations.

In conclusion, it was found that PD confers a higher risk of dementia than the general population, especially in those aged $<70$ years in both sexes. Regular monitoring for the development of dementia in patients with PD longitudinally is recommended. Future research should include further evaluation of the underlying mechanism and subtypes for dementia development after diagnosis of PD.

Acknowledgements We would like to thank the Bureau of National Health Insurance in the Ministry of Health and Welfare and the National Health Research Institutes for providing the National Health Insurance Research Database used in this study.

Contributors C-CL analysed the data and wrote the draft of the manuscript. YS and $\mathrm{P}-\mathrm{CL}$ provided further data analyses and interpretation. $\mathrm{C}-\mathrm{YL}$ and $\mathrm{SCH}$ advised the study and revised the manuscript. All authors have approved the final version of the manuscript.

Funding This study was supported by a grant from Taiwan Ministry of Science and Technology (MOST 106-2314-B-227-010).

Disclaimer The interpretation and conclusions contained herein do not represent those of the Bureau of National Health Insurance, Ministry of Health and Welfare, or National Health Research Institutes.

Competing interests None declared.

Patient consent for publication Not required.

Ethics approval $A$ full review by the institutional review board was not required because the encryption of the identification numbers makes it impossible to identify individuals. Access to the National Health Insurance Research Database datasets is approved by the National Health Research Institutes Review Committee.

Provenance and peer review Not commissioned; externally peer reviewed.

Data sharing statement We, as the authors of this original research article, state that there is no additional, unpublished data available from this study. Raw data sharing from National Health Insurance Research Database is prohibited according to the National Health Research Institutes policies in Taiwan.

Open access This is an open access article distributed in accordance with the Creative Commons Attribution Non Commercial (CC BY-NC 4.0) license, which permits others to distribute, remix, adapt, build upon this work non-commercially, and license their derivative works on different terms, provided the original work is properly cited, appropriate credit is given, any changes made indicated, and the use is non-commercial. See: http://creativecommons.org/licenses/by-nc/4.0/.

\section{REFERENCES}

1. World Health Organization. Dementia: a public health priority. 2012 http://www.who.int/mental_health/publications/dementia_report_ 2012/en/ (Cited 2 Apr 2018).

2. Alzheimer's Disease International. World Alzheimer Report 2015: the global impact of Dementia. 2015 https://www.alz.co.uk/research/ world-report-2015 (Cited 1 Apr 2018).

3. Russell A, Drozdova A, Wang W, et al. The impact of dementia development concurrent with Parkinson's disease: a new perspective. CNS Neurol Disord Drug Targets 2014;13:1160-8.

4. Kalia LV, Lang AE. Parkinson's disease. The Lancet 2015;386:896-912.

5. Xu Y, Yang J, Shang $\mathrm{H}$. Meta-analysis of risk factors for Parkinson's disease dementia. Trans/ Neurodegener 2016;5:11.

6. Domellöf ME, Ekman U, Forsgren L, et al. Cognitive function in the early phase of Parkinson's disease, a five-year follow-up. Acta Neurol Scand 2015;132:79-88.

7. Anang JB, Gagnon JF, Bertrand JA, et al. Predictors of dementia in Parkinson disease: a prospective cohort study. Neurology 2014;83:1253-60.

8. Zhu K, van Hilten JJ, Marinus J. Predictors of dementia in Parkinson's disease; findings from a 5-year prospective study using the SCOPA-COG. Parkinsonism Relat Disord 2014;20:980-5.

9. Williams-Gray CH, Mason SL, Evans JR, et al. The CamPalGN study of Parkinson's disease: 10-year outlook in an incident populationbased cohort. J Neurol Neurosurg Psychiatry 2013;84:1258-64.

10. Aarsland D, Andersen K, Larsen JP, et al. Risk of dementia in Parkinson's disease: a community-based, prospective study. Neurology 2001;56:730-6. 
11. Perez F, Helmer C, Foubert-Samier A, et al. Risk of dementia in an elderly population of Parkinson's disease patients: a 15-year population-based study. Alzheimers Dement 2012;8:463-9.

12. Marder $\mathrm{K}$, Tang MX, Cote $\mathrm{L}$, et al. The frequency and associated risk factors for dementia in patients with Parkinson's disease. Arch Neurol 1995;52:695-701.

13. Levy G, Schupf N, Tang MX, et al. Combined effect of age and severity on the risk of dementia in Parkinson's disease. Ann Neurol 2002;51:722-9.

14. de Lau LM, Schipper CM, Hofman A, et al. Prognosis of Parkinson disease: risk of dementia and mortality: the Rotterdam Study. Arch Neurol 2005;62:1265-9.

15. Hobson P, Meara J. Risk and incidence of dementia in a cohort of older subjects with Parkinson's disease in the United Kingdom. Mov Disord 2004;19:1043-9.

16. Haugarvoll K, Aarsland D, Wentzel-Larsen T, et al. The influence of cerebrovascular risk factors on incident dementia in patients with Parkinson's disease. Acta Neurol Scand 2005;112:386-90.

17. Levy G, Tang MX, Cote LJ, et al. Do risk factors for Alzheimer's disease predict dementia in Parkinson's disease? An exploratory study. Mov Disord 2002;17:250-7.

18. Breteler MM, de Groot RR, van Romunde LK, et al. Risk of dementia in patients with Parkinson's disease, epilepsy, and severe head trauma: a register-based follow-up study. Am J Epidemiol 1995;142:1300-5.

19. Hely MA, Reid WG, Adena MA, et al. The Sydney multicenter study of Parkinson's disease: the inevitability of dementia at 20 years. Mov Disord 2008;23:837-44.

20. Kwon KY, Kang SH, Kim M, et al. Nonmotor symptoms and cognitive decline in de novo Parkinson's Disease. Can J Neurol Sci 2014;41:597-602.

21. Auyeung M, Tsoi TH, Mok V, et al. Ten year survival and outcomes in a prospective cohort of new onset Chinese Parkinson's disease patients. J Neurol Neurosurg Psychiatry 2012;83:607-11.

22. Huang YC, Wu ST, Lin JJ, et al. Prevalence and risk factors of cognitive impairment in Parkinson disease: a population-based casecontrol study in Taiwan. Medicine 2015;94:e782.

23. Lee SY, Ryu HJ, Seo JW, et al. Dementia-free survival and risk factors for Dementia in a Hospital-Based Korean Parkinson's Disease Cohort. J Clin Neurol 2017;13:21-6.

24. National Health Insurance Administration. Universal Health Coverage in Taiwan. 2017 https://www.nhi.gov.tw/English/Content_List.aspx? $\mathrm{n}=8$ FC0974BBFEFA56D\&topn=ED4A30E51A609E49 (Cited 8 May 2018).

25. Sun Y, Chang YH, Chen HF, et al. Risk of Parkinson disease onset in patients with diabetes: a 9-year population-based cohort study with age and sex stratifications. Diabetes Care 2012;35:1047-9.
26. Shen CC, Tsai SJ, Perng CL, et al. Risk of Parkinson disease after depression: a nationwide population-based study. Neurology 2013;81:1538-44.

27. Chiang CJ, Yip PK, Wu SC, et al. Midlife risk factors for subtypes of dementia: a nested case-control study in Taiwan. Am J Geriatr Psychiatry 2007;15:762-71.

28. Liu CC, Li CY, Lee PC, et al. Variations in Incidence and Prevalence of Parkinson's Disease in Taiwan: a population-based nationwide study. Parkinsons Dis 2016;2016:8756359.

29. Charlson ME, Pompei $P$, Ales KL, et al. A new method of classifying prognostic comorbidity in longitudinal studies: development and validation. J Chronic Dis 1987;40:373-83.

30. Charlson M, Szatrowski TP, Peterson J, et al. Validation of a combined comorbidity index. J Clin Epidemiol 1994;47:1245-51.

31. Driver JA, Kurth T, Buring JE, et al. Parkinson disease and risk of mortality: a prospective comorbidity-matched cohort study. Neurology 2008;70:1423-30.

32. McKeith IG, Boeve BF, Dickson DW, et al. Diagnosis and management of dementia with Lewy bodies: Fourth consensus report of the DLB Consortium. Neurology 2017;89:88-100.

33. Noordzij M, Leffondré K, van Stralen KJ, et al. When do we need competing risks methods for survival analysis in nephrology? Nephrol Dial Transplant 2013;28:2670-7.

34. Prentice RL, Kalbfleisch JD, Peterson AV, et al. The analysis of failure times in the presence of competing risks. Biometrics 1978;34:541-54

35. Braak H, Del Tredici K, Bratzke $\mathrm{H}$, et al. Staging of the intracerebra inclusion body pathology associated with idiopathic Parkinson's disease (preclinical and clinical stages). J Neurol 2002;249:1-5.

36. Braak H, Del Tredici K, Rüb U, et al. Staging of brain pathology related to sporadic Parkinson's disease. Neurobiol Aging 2003;24:197-211.

37. Matthews FE, Stephan BC, Robinson L, et al. A two decade dementia incidence comparison from the Cognitive Function and Ageing Studies I and II. Nat Commun 2016;7:11398.

38. Solomon A, Mangialasche F, Richard E, et al. Advances in the prevention of Alzheimer's disease and dementia. J Intern Med 2014;275:229-50.

39. Levy G, Tang MX, Cote LJ, et al. Motor impairment in PD: relationship to incident dementia and age. Neurology 2000;55:539-44.

40. de la Torre JC. Cardiovascular risk factors promote brain hypoperfusion leading to cognitive decline and dementia. Cardiovasc Psychiatry Neurol 2012;2012:1-15.

41. Liu $H$, Xing $A$, Wang $X$, et al. Regulation of $\beta$-amyloid level in the brain of rats with cerebrovascular hypoperfusion. Neurobiol Aging 2012;33:e31-42

42. Grant I, Heaton RK, McSweeny AJ, et al. Neuropsychologic findings in hypoxemic chronic obstructive pulmonary disease. Arch Intern Med 1982;142:1470-6. 